\title{
Identificação de danos estruturais empregando transformações integrais e o algoritmo de Luus-Jaakola
}

Luciano dos Santos Rangel ${ }^{1}$

Carlos Eduardo Zavoli Cordeiro ${ }^{2}$

Gustavo Nogueira Lopes da Silva ${ }^{3}$

Isabela Cristina da Silveira e Silva Rangel ${ }^{4}$

Diego Campos Knupp ${ }^{5}$

Leonardo Tavares Stutz ${ }^{6}$

Programa de Pós-Graduação em Modelagem Computacional, Instituto Politécnico, Universidade do Estado do Rio de Janeiro, Nova Friburgo, RJ

Resumo. O presente trabalho aborda a identificação de danos estruturais em uma viga de Euler-Bernoulli simplesmente apoiada. A modelagem matemática da estrutura pode ser dada por um problema de valor inicial, uma Equação Diferencial Parcial (EDP). Para resolver a EDP que modela a viga, este trabalho propõe a utilização da Técnica da Transformada Integral Generalizada (GITT) . A identificação de danos é modelada a partir de uma formulação de problemas inversos, buscando-se estimar um determinado campo de coesão baseado na resposta impulsiva da estrutura, onde o problema de minimização é resolvido pelo algoritmo Luus-Jaakola. Com os resultados obtidos, pode-se concluir que a estratégia adotada foi capaz de identificar os danos com acurácia.

Palavras-chave. Identificação de Danos, Transformada Integral Generalizada, Resposta Impulsiva.

\section{Introdução}

A identificação de danos estruturais é uma questão de fundamental importância na engenharia, visto que uma estrutura está sujeita a processos de deterioração e a ocorrência de danos durante a sua vida útil. A presença de danos compromete o desempenho e a integridade estrutural, podendo colocar vidas humanas em risco e resultar em perdas econômicas consideráveis. Por estes motivos, estudos envolvendo a identificação de danos estruturais a partir de alterações nas características de vibração da estrutura, são crescentes na literatura especializada [4].

\footnotetext{
${ }^{1}$ lrangel@iprj.uerj.br

${ }^{2}$ caduzavoli1@gmail.com

${ }^{3}$ gustavonogueiralopes@gmail.com

${ }^{4}$ isilva@iprj.uerj.br

${ }^{5}$ diegoknupp@iprj.uerj.br

${ }^{6}$ ltstutz@iprj.uerj.br
} 
O presente trabalho aborda o problema de identificação de danos em uma viga de Euler-Bernoulli simplesmente apoiada. O comportamento dinâmico do sistema é descrito por uma equação diferencial parcial, cuja solução analítica pode ser extremamente custosa ou até mesmo inexistente, dependendo do modelo de dano e da excitação externa considerados. Para contornar essas dificuldades, o presente trabalho propõe a utilização da Técnica da Transformada Integral Generalizada (GITT) para a obtenção da resposta da estrutura.

Um problema inverso de identificação de danos, formulado a partir da GITT, é aqui proposto como um problema de otimização, onde a integridade estrutural é descrita por um parâmetro de coesão [5].

Para a resolução do problema, foi considerado o método estocástico de otimização Luus-Jaakola [2]. Resultados numéricos, relativos ao problema de identificação de danos na presença de dados corrompidos com ruído, mostram a potencialidade do método proposto.

O trabalho é organizado como se segue. Na Seção 2 é apresentada a formulação matemática da viga de Euler - Bernoulli simplesmente apoiada, além da GITT. De forma sucinta, é apresentada a formulação do problema inverso, na Seção 3. Na Seção 4 são apresentados os resultados numéricos da identificação de danos e por último, são apresentadas as conclusões obtidas com a presente pesquisa e são realizadas algumas sugestões para trabalhos futuros.

\section{Formulação matemática e metodologia de solução}

A equação de movimento de uma viga de Euler-Bernoulli é dada por uma equação diferencial parcial, onde $w(x, t)$ representa a deflexão transversal em um ponto $x$ ao longo da viga, no instante de tempo $t$. A equação é descrita por

$$
\mu \frac{\partial^{2} w(x, t)}{\partial t^{2}}+\eta \frac{\partial w(x, t)}{\partial t}+\frac{\partial^{2}}{\partial x^{2}}\left[E I(x) \frac{\partial^{2} w(x, t)}{\partial x^{2}}\right]=f(x, t)
$$

sendo $\mu$ a massa por unidade de comprimento; $\eta$ o coeficiente de amortecimento viscoso; $E I(x)$ a rigidez à deflexão, onde $E$ é o módulo de elasticidade e $I$ é o momento de inércia de área da seção transversal da viga; e $f(x, t)$ representa um carregamento externo por unidade de comprimento.

No presente trabalho, a integridade estrutural é descrita pelo parâmetro de coesão $\beta$ definido como

$$
\beta(x)=E I(x) / E_{0} I_{0},
$$

onde $E_{0}$ e $I_{0}$ representam os valores nominais do módulo de elasticidade e do momento de inércia de área. Cumpre destacar que, $\beta=1$ representa ausência de dano, enquanto que $\beta=0$ representa uma ruptura local.

A deflexão transversal é submetida às seguintes condições de contorno nos suportes $x=0$ e $x=L$ e condições iniciais, dadas por:

$$
w(0, t)=0, \quad w(L, t)=0,\left.\quad E I(x) \frac{\partial^{2} w(x, t)}{\partial x^{2}}\right|_{x=0}=0,\left.\quad E I(x) \frac{\partial^{2} w(x, t)}{\partial x^{2}}\right|_{x=L}=0,
$$




$$
w(x, 0)=0,\left.\quad \frac{\partial w(x, t)}{\partial t}\right|_{t=0}=0 .
$$

Neste trabalho, a solução das Eqs. (1-4) é obtida por meio do método híbrido numéricoanalítico conhecido como Técnica da Transformada Integral Generalizada (GITT - Generalized Integral Transform Technique). A GITT é um método baseado em transformações integrais, sendo uma versão híbrida e mais sofisticada da Técnica da Transformada Integral Clássica (CITT - Classical Integral Transform Technique), permitindo o tratamento de problemas não-transformáveis a priori, incluindo problemas não-lineares [1]. Embora sua utilização tenha se difundido principalmente em aplicações de transferência de calor e escoamento, mais recentemente o método também foi utilizado na análise dinâmica de estruturas [3].

A partir da seguinte igualdade

$$
E_{0} I_{0} \frac{\partial^{2}}{\partial x^{2}}\left[\beta(x) \frac{\partial^{2} w}{\partial x^{2}}\right]=E_{0} I_{0} \frac{\partial^{4} w}{\partial w^{4}}+E_{0} I_{0} \frac{\partial^{2}}{\partial x^{2}}\left[(\beta(x)-1) \frac{\partial^{2} w}{\partial x^{2}}\right]
$$

a Eq. (1) pode ser reescrita como

$$
\mu \frac{\partial^{2} w}{\partial t^{2}}+\eta \frac{\partial w}{\partial t}+E_{0} I_{0} \frac{\partial^{4} w}{\partial w^{4}}=E_{0} I_{0} \frac{\partial^{2}}{\partial x^{2}}\left[(1-\beta(x)) \frac{\partial^{2} w}{\partial x^{2}}\right]+f(x, t) .
$$

Seguindo o formalismo para a solução via GITT, define-se o seguinte par de transformação integral

$$
\text { (a) } w(x, t)=\sum_{i=1}^{N_{t r}} \tilde{\psi}_{i}(x) \bar{w}_{i}(t), \quad(b) \bar{w}_{i}(t)=\int_{0}^{L} \tilde{\psi}_{i}(x) w(x, t) d x,
$$

sendo $\tilde{\psi}_{i}(x)$ e $\bar{w}_{i}(t)$ as autofuções normalizadas e os coeficientes de expansão relacionados às coordenadas modais, respectivamente, e $N_{t r}$ a ordem de truncamento da expansão.

As autofunções normalizadas são definidas como

$$
\tilde{\psi}_{i}(x)=\frac{\psi_{i}(x)}{N_{i}^{1 / 2}}, \quad N_{i}=\int_{0}^{L}\left[\psi_{i}(x)\right]^{2} d x .
$$

As autofunções $\psi_{i}(x)$ e os respectivos autovalores $\lambda_{i}$ são obtidos do seguinte problema de autovalor diferencial:

$$
E_{0} I_{0} \frac{\partial^{4} \psi_{i}(x)}{\partial x^{4}}-\lambda_{i}^{2} \mu \psi_{i}(x)=0, \quad \psi_{i}(0)=\psi_{i}(L)=\psi_{i}^{\prime \prime}(0)=\psi_{i}^{\prime \prime}(L)=0,
$$

que possui solução analítica explícita dada por

$$
\lambda_{i}=\sqrt{\frac{E_{0} I_{0}}{\mu L^{4}}} i^{2} \pi^{2}, \quad \psi_{i}(x)=\sin \left(\frac{i \pi}{L} x\right) .
$$

Aplicando-se o operador $\int_{0}^{L} \tilde{\psi}_{i}(x)(\bullet) d x$ na Eq. (6), obtém-se 


$$
\mu \frac{d^{2} \bar{w}_{i}(t)}{d t^{2}}+\eta \frac{d \bar{w}_{i}(t)}{d t}+\lambda_{i}^{2} \bar{w}_{i}(t)=\underbrace{\int_{0}^{L} \tilde{\psi}_{i}(x) E_{0} I_{0} \frac{\partial^{2}}{\partial x^{2}}\left[(1-\beta(x)) \frac{\partial^{2} w}{\partial x^{2}}\right] d x}_{\bar{g}_{i, 1}(t, \overline{\mathbf{w}})}+\underbrace{\int_{0}^{L} \tilde{\psi}_{i}(x) f(x, t) d x}_{\bar{g}_{i, 2}(t)},
$$

onde $\overline{\mathbf{w}}=\left\{\bar{w}_{1}(t), \bar{w}_{2}(t), \ldots, \bar{w}_{N_{t r}}(t)\right\}$ e $i=1,2,3, \ldots, N_{t r}$ o índice associado a coordenada modal captado pela solução. Integrando duas vezes por partes $\bar{g}_{i, 1}(t, \overline{\mathbf{w}})$, obtém-se

$$
\bar{g}_{i, 1}(t, \overline{\mathbf{w}})=E_{0} I_{0} \int_{0}^{L}(1-\beta(x)) \frac{d^{2} \tilde{\psi}_{i}(x)}{d x^{2}} \frac{d^{2} w}{d x^{2}} d x .
$$

Substituindo a fórmula da inversa, Eq. (7 a) na Eq. (12) obtém-se

$$
\begin{aligned}
& \bar{g}_{i, 1}(t, \overline{\mathbf{w}})=E_{0} I_{0} \int_{0}^{L}(1-\beta(x)) \frac{d^{2} \tilde{\psi}_{i}(x)}{d x^{2}} \sum_{j=1}^{N_{t r}} \frac{d^{2} \tilde{\psi}_{j}(x)}{d x^{2}} \bar{w}_{j}(t) d x= \\
& =E_{0} I_{0} \sum_{j=1}^{N_{t r}} \bar{w}_{j}(t) \underbrace{\int_{0}^{L}(1-\beta(x)) \frac{d^{2} \tilde{\psi}_{i}(x)}{d x^{2}} \frac{d^{2} \tilde{\psi}_{j}(x)}{d x^{2}} d x}_{A_{i, j}}=E_{0} I_{0} \sum_{j=1}^{N_{t r}} \bar{w}_{j}(t) A_{i, j} .
\end{aligned}
$$

Reescrevemos o problema transformado como

$$
\begin{gathered}
\mu \frac{d^{2} \bar{w}_{i}(t)}{d t^{2}}+\eta \frac{d \bar{w}_{i}(t)}{d t}+\lambda_{i}^{2} \bar{w}_{i}(t)=\bar{g}_{i, 1}(t, \overline{\mathbf{w}})+\bar{g}_{i, 2}(t), \\
\bar{w}_{i}(0)=0,\left.\quad \frac{d \bar{w}_{i}(t)}{d t}\right|_{t=0}=0 .
\end{gathered}
$$

Uma vez que o sistema de equações diferenciais ordinárias dado pelas Eqs. (14) e (15) é resolvido, a fórmula da inversa, Eq. (7 a) pode ser prontamente utilizada para obter uma expressão para a resposta $w(x, t)$. O sistema acoplado dado pelas Eqs. (14) e (15) não possui solução analítica explícita, e é então resolvido numericamente neste trabalho por meio da rotina intrínseca NDSolve, na plataforma Wolfram Mathematica, com controle automático de erro absoluto e relativo.

\section{Problema Inverso}

No presente trabalho, para a formulação do problema de identificação de danos no domínio do tempo, define-se o vetor de resposta generalizada

$$
\mathbf{z}_{E}=\left[\begin{array}{llll}
z_{E}\left(t_{1}\right) & z_{E}\left(t_{2}\right) & \ldots & z_{E}\left(t_{N}\right)
\end{array}\right]^{T},
$$

onde $z_{E}\left(t_{i}\right)$ representa a resposta experimental da estrutura, amostrada no instante de tempo $t_{i}$ e $N$ é o número total de amostras consideradas. 
O problema de identificação de danos é, então, definido como

$$
\min _{\boldsymbol{\beta}} \frac{1}{2}\left\|\mathbf{z}_{E}-\mathbf{z}(\boldsymbol{\beta})\right\|^{2}
$$

onde $\mathbf{z}(\boldsymbol{\beta})$ corresponde ao vetor de resposta generalizada previsto pelo modelo da estrutura. No presente trabalho, para a resolução do problema inverso definido na Eq. (17), utiliza-se o método estocástico de otimização Luus-Jaakola [2].

\section{Resultados Numéricos}

O trabalho considera uma viga de Euler-Bernoulli simplesmente apoiada, cujos parâmetros físicos são: $E_{0} I_{0}=3,5 \mathrm{Nm}^{2}, L=1,0 \mathrm{~m}, \mu=0,4 \mathrm{~kg} \cdot \mathrm{m}^{-1}$ e $\eta=5,0 \mathrm{Ns}^{-m^{-2}}$. O campo de coesão foi discretizado em 21 nós, resultando 21 parâmetros nodais de coesão, e o deslocamento transversal da viga foi considerado como medido em $x_{0}=0,2 \mathrm{~m}$, onde também se deu a excitação do tipo impulso unitário, dada por

$$
f(x, t)=\delta_{t}(t) \delta_{x}\left(x-x_{0}\right),
$$

onde

$$
\delta_{t}(t)=\left\{\begin{array}{l}
\frac{1}{c}, \text { se } t \leq c \\
0, \text { se } t>c
\end{array}\right.
$$

com $c$ igual a $10^{3}$ e

$$
\delta_{x}\left(x-x_{0}\right)=\left\{\begin{array}{l}
0, \text { se } x<x_{0}-\frac{g}{2} \\
\frac{1}{g}, \text { se } x_{0}-\frac{g}{2} \leq x \leq x_{0}+\frac{g}{2} \\
0, \text { se } x<x_{0}+\frac{g}{2}
\end{array}\right.
$$

com $g$ igual a $10^{-2}$ e $x_{0}$ é a posição da excitação.

Para obtenção da resposta experimental sintética, considerou a adição de ruído de distribuição gaussiana, de média nula e desvio padrão determinado indiretamente pela razão-sinal-ruído (SNR), definida como

$$
\mathrm{SNR}=10 \log \left(P_{s} / P_{r}\right),
$$

onde $P_{s}$ e $P_{r}$ são, respectivamente, a potência do sinal e do ruído. Nos casos simulados, foi considerado, aproximadamente, um nível de ruído de $16 \mathrm{~dB}$.

A imposição do dano à viga, em uma dada posição $x$, foi realizada alterando-se a espessura relativa $h(x) / h_{0}$ da seção transversal da mesma, onde $h_{0}$ é a espessura nominal. Foram considerados dois cenários de dano. No primeiro, foi considerado um campo de dano simples com redução na altura relativa de $10 \%$ na posição $0,4 \mathrm{~m}$. No segundo caso, foi considerado um campo de dano com redução de $20 \%$ na altura relativa nas posições 0,45 m e 0,75 m. O resultado da identificação de danos para os dois casos é apresentado na Figura 1. 


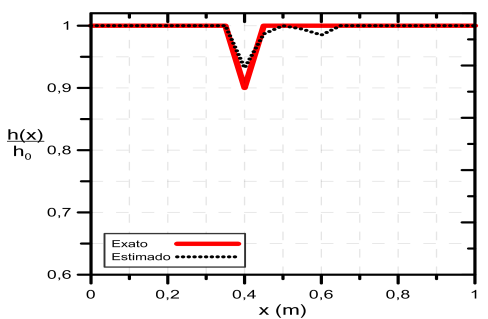

(a) Caso 1

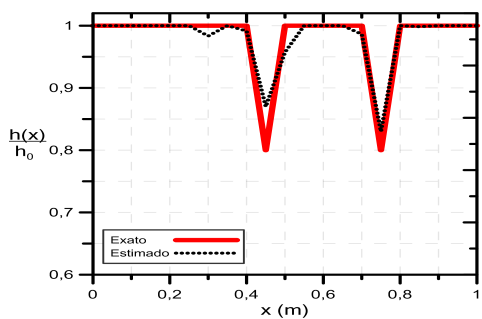

(b) Caso 2

Figura 1: Identificação de danos considerando o método Luus-Jaakola.

Com pode ser observado na Figura 1, o método de identificação de danos proposto foi capaz de identificar os cenários de dano considerados, mesmo na presença de ruído de intensidade considerável.

\section{Conclusões}

No presente trabalho, um problema inverso de identificação de danos estruturais, em uma viga de Euler-Bernoulli, foi formulado a partir da Técnica da Transformada Integral Generalizada.

Para a resolução do problema inverso de identificação de danos, foi considerado o método de otimização de Luus-Jaakola. O método proposto foi capaz de identificar os campos de dano considerados, mesmo na presença de elevado nível de ruído.

Perante os resultados, pode-se concluir que a estratégia utilizada conseguiu recuperar de forma satisfatória, a localização e intensidade dos danos, mesmo considerando ruído aditivo de elevada intensidade.

Como sugestões para trabalhos futuros, o mesmo problema será abordado considerando a combinação da Transformada Integral Clássica e a GITT; e será considerada também a abordagem bayesiana na formulação do problema inverso.

\section{Agradecimentos}

Os autores deixam aqui registrados os agradecimentos às agências de fomento CAPES, CNPq e FAPERJ pelo apoio concedido.

\section{Referências}

[1] R.M. Cotta. Integral Transforms in Computational Heat and Fluid Flow. CRC Press, pages 158, 1993.

[2] R. Luus, T. H. I. Jaakola. Optimization by Direct Search and Systematic Reduction of the Size of Search Region. AIChE Journal, Issue 4,19: 760-766, 1973. 
[3] C. F. T. Matt. Combined classical and generalized integral transform approaches for the analysis of the dynamic behavior of a damaged structure. Applied Mathematical Modelling, 37: 8431-8450, 2013.

[4] I. C. S. S. Rangel. Identificação de danos estruturais a partir do modelo de superfície de resposta. Dissertação de Mestrado em Modelagem Computacional, Instituto Politécnico, Universidade do Estado do Rio de Janeiro, Nova Friburgo, pages 109, 2014.

[5] L. T. Stutz, D. A. Castello, F. A. Rochinha, A flexibility-based continuum damage identification approach. Journal of Sound and Vibration, 279: 641-667, 2005. 